\title{
Research on Electric Automation and Energy Saving Control Technology
}

\author{
Hanjie Sun ${ }^{1, a}$ \\ ${ }^{1}$ Shandong University of Science and Technology, Jinan, Shandong, 250031 \\ ${ }^{a}$ email
}

Keywords: Electric Automation, Energy Saving, Control Technology

\begin{abstract}
Automation, information, intelligent and digital way will be in all areas of society attention, especially in the field of electrical energy automation control, it is China's economic development plays a decisive role. Electric energy-saving design technology to China's power industry has brought a substantial increase in efficiency, but also for the current electrical automation of energy-saving design technology status, and strive to improve the imperfect areas, in order to achieve the depth of automation and control technology to speed up The speed of development of automated control technology.
\end{abstract}

\section{Introduction}

Electrical automation energy-saving system design is in the design of electrical automation system, from the energy saving and consumption direction to the corresponding design work, through the rational use of harmonic elimination technology, optimize the power transmission distance, modify the power distribution, change the power grid material, New transformers and other measures, the use of various means to re-design and deployment of electrical automation systems, electrical automation system to achieve energy efficiency and objectives of the guarantee.

The trend of electrical automation in the artificial intelligence, expert systems, broadband communications, fuzzy control technology and other automation technology based on the development of the network is showing a digital, digital features. The use of electrical automation systems, technical advantages, to achieve electrical energy and power reduction has become an effective weapon industry. The new period, from the global pattern, who can deepen the development of energy-saving energy trends, who will be able to grasp the development of the world power industry opportunities and initiative.

\section{Electric energy saving automation control system}

Electric energy-saving automatic control system is composed of motor and power supply, detection, control device composed of feedback control system, can automatically complete the energy conversion and control the necessary information processing. The electric energy saving automatic control system has the control device which may turn on or off the circuit according to the external signal request, change the parameter, realize the control circuit to the circuit or the non-electric parameter. Mainly in: the DC system and LPS system monitoring; control and operation of the generator and security power supply; control and operation of the transformer; for the $380 \mathrm{~V}$ low-voltage plant monitoring and operation; on the excitation, demagnetization operation, control mode switch; On the variable group excitation transformer protection and control functions. Electric energy-saving automation systems are often have a simple operation of the control panel through the button to operate, electrical energy-saving automation equipment often work on the dynamic information real-time tracking records, and do a certain storage records, you can make a certain correlation report. Electric energy-saving automation control often through the frequency control, direct start and star or triangular start control, so that can ensure the stable operation of production equipment, if found to be wrong, the operator will be able to immediately make chain control. The range of energy-efficient design technologies for electrical automation is extensive, such as industry, agriculture, and defense. The healthy development of energy-saving design technology of electric 
automation will make our country's economic development have a greater breakthrough, increasingly meet people's requirements for electrical energy. In order to meet the requirements of the times many colleges and universities have also set up the electrical automation of energy-saving design technology, professional knowledge and real life are closely linked, recently learn more and more automated control technology, Get gradually improved. The development of energy-saving design of electrical automation is conducive to improving people's living standards, while promoting the development of China's economy.

\section{The technical focus of energy - saving design in the process of electrical automation under the new situation}

The premise of electrical automation is the high efficiency of energy, low pollution transmission, and according to the laws of physics, energy in the transmission process will be due to resistance, distance and other factors to produce a certain loss in the construction of electrical automation should be Control the transmission loss as a key technical aspects, by reducing the transmission loss and improve the efficiency of power transmission, reduce transmission costs, control the possible pollution and hazards. On the one hand, the electrical automation process to use benign alloy material as a wire material, which can reduce the power transmission and use of the process of loss, control the entire electrical automation system resistance loss. On the other hand, the process of electrical automation to optimize the wiring of the wire form, by reducing the length of the wire, optimize the power supply distance, change the load distribution form and other technical means to control the power in the electrical automation system in the loss and waste. In addition, according to the physical formula, you can increase the electrical cross-section of the electrical equipment to reduce the resistance of the entire system, and thus achieve the effective control of energy consumption during transmission.

The process of electrical automation requires the application of a large number of different types of transformers, and the realization of electrical automation energy-saving targets also need to scientifically set, select and apply the transformer. On the one hand, in the construction of electrical automation to energy-saving transformers as the preferred target, in order to reduce the active loss from the transformer to achieve energy-saving electrical automation development goals. On the other hand, to ensure the balance of the three-phase current transformer, the process of electrical automation is not only a simple electrical mechanical addition, in the electrical automation system design and construction control transformer three-phase current balance, in optimizing the circuit load While controlling the transformer ontology energy consumption, from a larger system to achieve the goal of electrical automation and energy conservation. In addition, in the electrical automation system in the rational application of compensation transformers, so that power and energy consumption can be further optimized to avoid imbalance and imbalance problems arising from the emergence of excessive energy consumption problems.

Reactive power compensation is an important basic energy-saving technology and measures in the construction of electrical automation. Therefore, it is necessary to give reactive power compensation. In the process of electrical automation, reactive power compensation is an important basic energy-saving technology and measures. Therefore, The application of technology to a reasonable attention. In the application of reactive power compensation technology in the electrical automation system, we should pay attention to the following aspects: on the one hand, the capacitor should be selected when the capacity of the standard, to refer to the electrical power equipment, power factor, electrical automatic system capacity, electrical automatic system Load and other parameters, through scientific calculations to obtain accurate capacity standards. On the other hand, to choose a smart compensation equipment, to achieve smooth adjustment, the whole process of tracking and the full range of adaptation, in order to improve the compensation effect and quality, to achieve the goal of energy saving and stability of electrical safety assurance. In addition, in the reactive power compensation equipment design and installation process to "local compensation" as a basic principle, in reducing the network and system reactive power loss at the same time, to achieve the goal of energy-saving operation of the electrical automation system. 
In the electrical automation system, different equipment and complex operation will produce a threat and energy consumption of large harmonics, which will increase the electrical automation system instability and energy consumption, in order to achieve the basic goal of electrical automation and energy saving, Electrical automation system in the rational use of filtering technology to eliminate the operation, current oscillation, frequency changes arising from the harmful harmonics. In the process of selection of filtering technology should pay attention to the following points: On the one hand, in the electrical automation system to be the preferred active filter, active filter with dynamic stability, versatility, wide range of advantages, in the electrical automation system Can use the reactive power compensation measures to effectively suppress and eliminate the impact of harmonics, so that the electrical automation systems and equipment effective protection, and thus achieve energy-saving development. On the other hand, in the electrical automation system, the application of filtering technology to prevent the application of the main points, can be mistaken operation and equipment before the operation to start the filter function, which will ensure that the electrical automation function is achieved at the same time, to achieve electrical automation equipment Of energy-saving operation.

\section{Current Status and Development Trend of Electric Energy Saving Automation Control}

Current Situation of Automatic Control of Electric Energy Saving. Electric energy-saving automation control system mainly has centralized monitoring mode, remote monitoring mode and field bus monitoring mode. Centralized control mode is often easy to run and maintain, it is the system's various functions are concentrated to a processor, and then to deal with; remote monitoring, mainly with energy-saving cable, saving installation costs, high reliability, the main application In small system monitoring. Field bus monitoring methods are often more targeted for system design, this monitoring is often in addition to the advantages of remote monitoring, but also can significantly reduce the amount of work used. At present, the advantage of centralized control and centralized control is that it is easy to operate and maintain. The centralized control mode is to concentrate all the functions of the system into a processor and then process it.

Development Trend of Automatic Control of Electric Energy Saving. The development of energy-saving design technology of electrical automation is inextricably linked with its own characteristics. Before the immaturity of technology, the development of energy-saving design technology of electric automation has taken a detour, but the current energy-saving automation technology has already met the times Development, combined with a wide range of information technology applications to the actual production. Computer used in electrical energy conservation local or overall control, become the core areas of electrical energy automation control. As the development of science and technology more and more quickly, it drives the development of automation, after the virtual reality technology and video processing technology applications will be more extensive. Automation control system status will be greatly enhanced, such as computers, network technology and multimedia technology have a far-reaching application prospects.

In the process of running the power system, the reactive power in the power distribution equipment occupies a large capacity, so there is a situation of more serious energy consumption, which not only affects the voltage but also adversely affects the quality of the whole power. For the user, the reactive power is mainly expressed in low power, especially when the power is too low, the user needs to pay a certain amount of fines to the relevant departments, so if you can use the appropriate reactive power compensation equipment, you can guarantee the power Of the balance, effectively improve the quality of power supply. Staff in the operation to minimize the unnecessary loss of electricity, so as to ensure the safe operation of electrical engineering. If the wire is affected by the resistance, then the electrical energy generated by the motor can not be better absorbed by the transformer, while those who have not been absorbed in the process of current transmission will be released. At the same time due to reactive power produced a certain role, so you can let the capacitor and reactive power to offset. (1) the use of capacitors in the reactive power compensation, must be based on the voltage capacity, voltage load, natural power, the target power and other parameters to determine the capacitor (in the case of reactive power compensation) (2) to determine 
the parameters of the physical quantity of reactive current, power parameters, so as to effectively avoid the need for a series of quantitative reactors to eliminate harmonics; Switching phenomenon.

\section{Conclusion}

With the rapid development of modern science and technology, human society has entered the information age today. The rapid development of science and technology has promoted the development of society towards informationization and automation. In order to improve the current situation of energy-saving design technology of electric automation, Automation control technology to improve the speed of automation and control technology to speed up the development.

\section{References}

[1] Jin Lihong. Explore electrical automation in commercial building central air conditioning in the realization of energy [J]. Fujian Building Materials. 2015 (04)

[2] Jia Haiyan. High-rise building central air conditioning electrical automation control technology analysis [J]. Doors and windows. 2014 (03)

[3] Su Peng. On the application of electrical automation in the central air conditioning analysis [J]. Henan Science and Technology. 2014 (06)

[4] Li Chaobing. Electrical automation in the central air conditioning in the practical application [J]. Technology entrepreneurs. 2013 (20)

[5] Shang Liangjun, Du Honglei. Talking about the application of industrial electrical automation in clean air conditioning system [J] .New Technology \& New Products. 2013 (11) 